\title{
Comparison Between Bivariate Phase-Rectified Signal Averaging and Sequence Method in Assessing the Baroreflex Sensitivity
}

\author{
Massimo W. Rivolta, Roberto Sassi \\ Dipartimento di Informatica, Università degli Studi di Milano, Milan, Italy
}

\begin{abstract}
Baroreflex sensitivity (BRS) measures the rate of changes in inter-beat time intervals $(R R)$ with respect to changes in blood pressure (BP). Recently, Bivariate PhaseRectified Signal Averaging (BPRSA) was proposed as possible BRS estimator and was compared with the validated sequence method (SM). However, the two methods differ substantially, questioning whether BPRSA might be considered as an estimator of BRS. In this study, we investigated the role that the coupling between $R R, B P$ and respiration has on BRS estimates provided by BPRSA and SM. Multivariate autoregressive models (MVAR) were fitted to the data of 10 healthy subjects that underwent a tilt test. MVAR models were then used to generate synthetic signals while artificially varying the coupling between $R R$, $B P$ and respiration. Both BPRSA and SM provided higher $B R S$ values during supine with respect to head-up phase. Computerized simulations showed little influence of the coupling between respiration and RR on both estimators, whereas a major difference appeared when the coupling between BP and RR was removed. Then, BPRSA almost vanished, while SM increased of about $10 \%$, regardless the phase. In conclusion, BRS estimates using BPRSA were highly dependent on the coupling between BP and $R R$, while SM resulted in more stable estimates.
\end{abstract}

\section{Introduction}

The baroreflex (BR) mechanism is responsible in maintaining homeostasis of blood pressure in response to internal and external perturbations, by modulating the heart rate. The rate of change between inter-beat time intervals (RR) and blood pressure, i.e., the BR sensitivity (BRS), was found clinically relevant (e.g., [1]). Numerous are the techniques aiming to quantify BRS, with the sequence method (SM) being one of the most used and validated algorithm. Briefly, SM identifies increasing (or decreasing) ramps, concurrently occurring in both RR and blood pressure series, and then it quantifies the slope between RR and blood pressure values belonging to the ramps.
Recently, Schumann et al. [2] proposed an extension of the Phase-Rectified Signal Averaging (PRSA) technique [3] capable to deal with two signals concurrently measured. This technique is called Bivariate PRSA (BPRSA) and splits the identification of the anchor points and the synchronous averaging on two different signals, i.e., anchor points are located on the trigger signal while the averaging is performed on the target one, synchronously to the anchor points. Bauer et al. [4] proposed to use BPRSA to quantify BRS from RR and systolic blood pressure (SBP) series and found that it was an independent predictor of death in a cohort of heart failure patients.

Despite the importance of the study, Castiglioni et al. [5] questioned whether what computed through BPRSA might be considered an estimated of BRS. The main two criticisms were the following. First, sensitivity is defined as ratio of the variations in RR and SBP, while BPRSA does not consider variations of the latter in the estimate of BRS. Second, the anchor points are solely defined on the SBP series, thus likely averaging RR values on events synchronous with the SBP but not related with the baroreflex.

Interestingly, Müller et al. [6] repeated the study of Bauer et al., after normalizing the BRS estimated, obtained through BPRSA, by the average increase in the SBP series at the anchor point. This normalization tackled properly the first criticism and obtained substantially the same predicting power in the same cohort.

In order to shed some light on the second criticism, we quantified the BRS using both SM and BPRSA on synthetic data generated by multivariate autoregressive (MVAR) models, comprising of RR, SBP and respiration series. The main rationale was that, as suggested by Castiglioni et al., a third component, i.e., the respiration, with different levels of coupling with RR and SBP, may alter what BPRSA captures with respect to SM. In addition, we studied the case resembling the lowest level of coupling possible, in which the RR and SBP were statistical independent, by quantifing the BRS through SM and BPRSA. In this regard, for one of the variants of SM, we derived a closed-form solution of the BRS, specifically when RR and SBP were modeled with two independent White Gaussian Noises (WGN). 


\section{Methods}

\subsection{BRS estimation using SM and BPRSA}

Both SM and BPRSA make use of two time series $x(k)$ (SBP, the trigger) and $y(k)(\mathrm{RR}$, the target), where $k$ is the time index.

Different implementations of the SM algorithm are available $[7,8]$. In this work, we used the global SM proposed in [8]. Briefly, we first identified all the ramps occurring in both trigger and target signals. Ramps were defined as strict increases. Second, from each sequence identified its average was subtracted. Third, the global BRS was computed as the slope estimated by all pairs of points $(x(k), y(k))$ with $k$ spanning all the samples belonging to the ramps. We restricted the computation only to ramps lasting for four samples.

The BPRSA algorithm is composed of two steps [2]. First, anchor points associated to signal increases are identified on the trigger series $x(k)$ and inserted in the anchor points' list. A time index $k$ is defined as anchor point when the sum $x(k)+\cdots+x(k+T-1)$ is larger than the sum $x(k-T)+\cdots+x(k-1)$. Second, all the windows of $2 L$ elements centered on each anchor point on the target series $y(k)$ are aligned (anchor points are located at the position of the $L+1$ sample) and then averaged. The wavelet transform of the BPRSA series is then used to quantify the average increase. Previous works [6,9] quantified BRS by using $T=1$ and a Haar wavelet of order 2, and normalized the quantity by the average SBP increase at the anchor point.

\subsection{Dataset and signal extraction}

We used data from the "Physiologic Response to Changes in Posture" dataset, freely available on Physionet $[10,11]$. Briefly, single lead ECG and continuous blood pressure from 10 healthy subjects were measured during a tilt test. The protocol was composed of six phases including two slow tilts, two rapid tilts, and two standingup maneuvers. Each phase lasted three minutes and was separated by the following one by five minutes of resting supine. The order of the phases was randomized.

In this study, we considered only signals in proximity to the first rapid tilt $\left(75^{\circ}\right.$ tilt in $\left.2 \mathrm{~s}\right)$ of each subject, since such tilt proved to cause evident variations on both heart rate and blood pressure [11]. In particular, we extracted two segments of two minutes. The first one was immediately prior to the rapid tilt while resting supine and the second one during the last two minutes after the rapid tilt.

Both RR and SBP series were extracted from the given signals (sampling rate of $250 \mathrm{~Hz}$ ). In particular, for the $k$ th beat, $\operatorname{RR}(k)$ was defined as the time interval between the $k$-th beat and its preceding one, while $\operatorname{SBP}(k)$ as the maximum blood pressure value identified in the same interval. RR values above $1500 \mathrm{~ms}$ were considered artifacts and substituted with linear interpolation.

From the single lead ECG available, we derived the respiration rate series $\operatorname{RESP}(k)$ using the procedure described in [12]. Briefly, the area of the each QRS complex in a $50 \mathrm{~ms}$ window centered on the $\mathrm{R}$ peak was computed for each beat. Then, an autoregressive (AR) model was fitted on this sequence on windows of $20 \mathrm{~s}$ with a step of $5 \mathrm{~s}$, and the pole associated the slowest rate and a certain minimum magnitude, located between 12 and 25 breaths per minute, was identified as respiration rate. Linear interpolation was used to have an estimate for each beat.

Very low frequency components were removed from the three series using a high pass filter (3rd Butterworth filter, zero phase, cut-offs: $0.04 \mathrm{~Hz}, 0.025 \mathrm{~Hz}$, and 12 breaths per minute for RR, SBP and RESP, respectively).

\subsection{Experiments}

Three experiments were carried out. First, we computed BRS using the two methodologies reported in sec. 2.1 on the dataset considered. The aim of this experiment was to verify that the change observed in blood pressure and heart rate from supine to head-up phase changed BRS as well. This verification was preparatory for the subsequent experiments.

Second, we investigated on the behavior of the two methodologies when applied to two independent WGN. To do so, we applied SM and BPRSA on two independent WGN having variances equal to those measured on the RR and SBP series of each subject. For the global SM, BRS was computed using the theoretical formulation derived in sec. 2.4, while for BRSA, two sequences of 100000 samples were generated and used to guarantee the convergence of the estimator.

In the third experiment, we quantified the percent variation of BRS after removing the coupling between i) SBP and RR; and ii) RESP and RR. With this goal, we modeled RR, SBP and RESP of each subject during supine and head-up using a MVAR model of order 5 [13], Then, we generated synthetic signals of 100000 samples in three different conditions: i) using the full MVAR model; ii) setting to zero the coefficients linking RR and SBP; and iii) setting to zero the coefficients linking RR and RESP. Series were rescaled to have the variance observed on the real data.

\subsection{Theoretical value of the global SM}

In this section, we derive the output of the global SM described in [8], applied to two independent WGN $x$ and $y$ for sequences of four consecutive samples. Such sequences can be modeled using two random vectors $X=$ $\left[x_{1}, x_{2}, x_{3}, x_{4}\right]^{\top}$ and $Y=\left[y_{1}, y_{2}, y_{3}, y_{4}\right]^{\top}$ with zero mean 


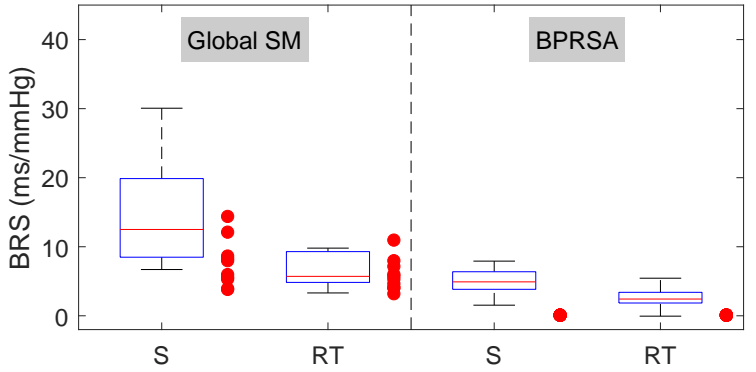

(a)

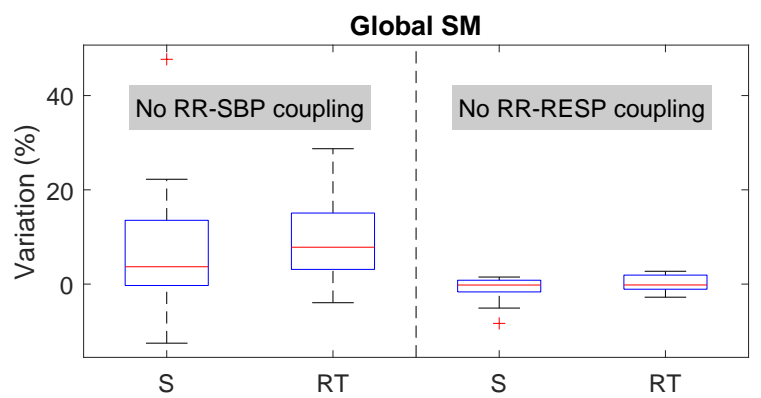

(c)

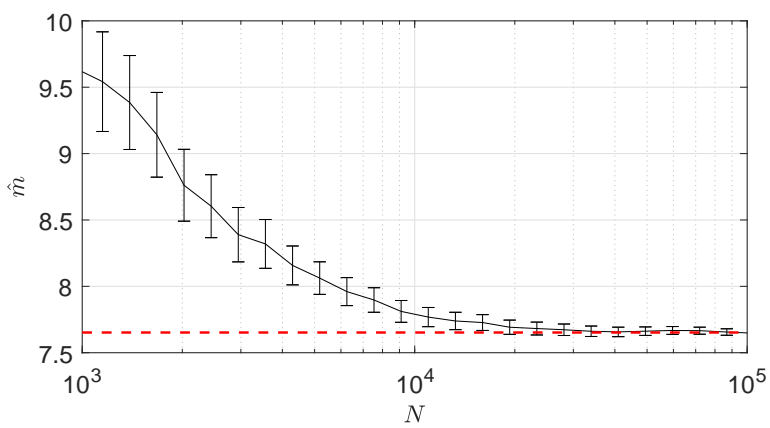

(b)

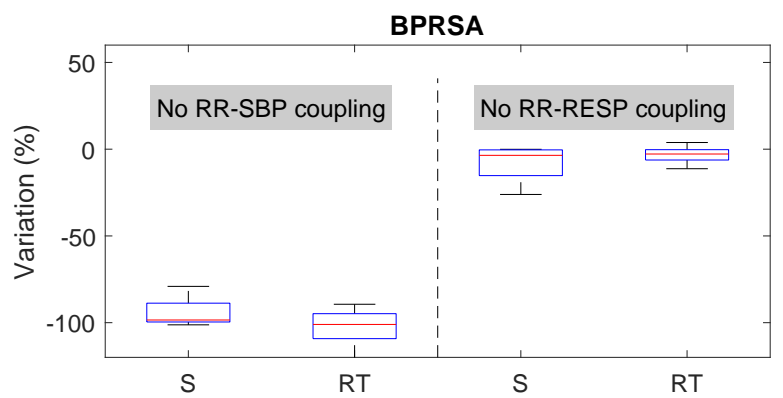

(d)

Figure 1: (a) BRS computed using the global SM and BPRSA. S and RT stand for resting supine prior the tilt and rapit tilt phase, respectively. Red dots refer to the BRS computed on WGN. Of note, dots for the global SM were computed using (2). (b) Average and $95^{\text {th }}$ confidence interval of the estimate $\hat{m}$ (black error bar) and the theoretical value $m$ (red dashed line), while varying the number of samples $N$ (logarithmic scale). Each error bar contains 1000 independent estimates. $\sigma_{x}=5$ and $\sigma_{y}=50$. (c) and (d) Percentage of variation on BRS for both global SM and BPRSA, when the coupling between SBP and RR, and RESP and RR were removed.

and standard deviations $\sigma_{x}$ and $\sigma_{y}$. The joint probability density function is a modified Multivariate Gaussian Distribution, adapted to handle ramps by restricting the space spanned by $(X, Y)$. In this particular condition, the joint probability becomes

$$
\begin{aligned}
& p\left(x_{1}, x_{2}, x_{3}, x_{4}, y_{1}, y_{2}, y_{3}, y_{4}\right)= \\
& =\frac{576}{\left(\sigma_{x} \sigma_{y}\right)^{4}} \prod_{i=1}^{4} \phi\left(\frac{x_{i}}{\sigma_{x}}\right) \phi\left(\frac{y_{i}}{\sigma_{y}}\right) \prod_{j=2}^{4} 1_{x_{j-1}>x_{j}} 1_{y_{j-1}>y_{j}}
\end{aligned}
$$

where $\phi(z)$ is the standard normal distribution, $1_{c}$ is the indicator function with a condition $c$, and the constant value 576 forces the area under $p$ to be 1 . Indeed, the probability of having a ramp on any of the two series was $1 / 24$. Therefore, the normalizing factor was $24^{2}=576$.

The global SM determines the slope of the line, estimated using the least square approach, from the scatter plot of all pairs $\left(x_{i}, y_{i}\right)$ for $i \in\{1,2,3,4\}$ associated to ramps, after removing their mean values. Least square estimation in the probabilistic form can be obtained by minimizing the following function

$$
\epsilon=\sum_{i=1}^{4} \int_{x_{i}} \int_{y_{i}}\left(\tilde{y}_{i}-m \tilde{x}_{i}-q\right)^{2} p d x_{i} d y_{i}
$$

where $m$ is the slope to be estimated, $q$ is the intercept of the linear model, $\tilde{x}_{i}=x_{i}-\frac{x_{1}+x_{2}+x_{3}+x_{4}}{4}, \tilde{y}_{i}=$ $y_{i}-\frac{y_{1}+y_{2}+y_{3}+y_{4}}{4}$, whereas $p$ is the joint probability density function of all the eight variables, respectively.

It is possible to show that the slope that minimizes (1) is

$$
m=\left(A^{2}+B^{2}\right)\left(\frac{\sigma_{y}}{\sigma_{x}}\right) \approx 0.7652\left(\frac{\sigma_{y}}{\sigma_{x}}\right)
$$

where $A=\frac{2 \sqrt{6}}{\pi \sqrt{\pi}} \tan ^{-1}(\sqrt{2})$ and $B=\frac{2 \sqrt{6}}{\sqrt{\pi}}-$ $\frac{6 \sqrt{6}}{\pi \sqrt{\pi}} \tan ^{-1}(\sqrt{2})$. Figure 1 reports an example of slope estimate $\hat{m}$ using the global SM and its theoretical value $m$ for $\sigma_{x}=5$ and $\sigma_{y}=50$, computed using (2), while varying the number of sample $N$. The intercept becomes $q=0$. 


\section{Results}

Global SM and BPRSA were able to distinguish the supine phase from the head-up one after rapid tilt (Fig. 1a), with higher BRS estimates during the former phase. BRS estimates obtained using the global SM were higher than those of BPRSA in both phases (Fig. 1a).

When global SM and BPRSA were applied to WGN, they led to different behaviors. On one hand, BPRSA estimates converged to zero (red dots in Fig. 1a). On the other hand, global SM values were proportional to the ratio of the standard deviations of RR and SBP, as predicted by (2).

The effect on BRS of the coupling between RR and SBP was larger with respect to the one between RR and RESP (Fig. 1c-d). For the former case, BRS computed using BPRSA almost vanished (Fig. 1d), while the global SM had an average increase of about $10 \%$. No substantial difference were found between supine and head-up phases for both global SM and BPRSA.

\section{Discussions}

BRS values during resting supine were found higher than those during head-up, as expected when using SM [5]. Interestingly, BRS estimated using BPRSA had the same trend but on a different scale (Fig. 1a). This effect might be what speculated by Castiglioni et al. regarding the work of Bauer et al., in which some physiological interactions, different from the baroreflex feedback (for instance, sympathetic activation, or, as in this case, respiration), might have changed between supine and head-up phases.

In order to shed light on these possible alternative interactions, we firstly investigated on the case in which RR and SBP were statistical independent. We found that BPRSA correctly estimated a zero BRS, whereas SM estimates were dependent on their standard deviations (red dots in Fig. 1a). This was due to the fact that some ramps were identified concurrently on RR and SBP just by chance (1 ramp every 576 samples, as predicted in sec. 2.4).

Then, we studied the influence of the coupling between $\mathrm{RR}, \mathrm{SBP}$ and respiration on BRS, using MVAR models. We found that both SM and BPRSA were little influenced by respiration and were similarly capable in distinguishing supine and head-up phases. This might be due to the predominant coupling between RR and SBP (Fig. 1c-d). On the other hand, when no coupling between RR and SBP was imposed, BPRSA almost converged to zero (Fig. 1d), while SM likely measured the influence of SBP on RR through respiration.

In conclusion, global SM and BPRSA seemed to carry similar information, likely due to the strong coupling between SBP and RR, while respiration played a little role. However, we do not exclude that other physiological inter- actions may produce differences between the estimates of the two methodologies.

\section{References}

[1] La Rovere MT, Bigger JTJ, Marcus FI, et al. Baroreflex sensitivity and heart-rate variability in prediction of total cardiac mortality after myocardial infarction. ATRAMI investigators. Lancet 1998;351(9101):478-484.

[2] Schumann AY, Kantelhardt JW, Bauer A, et al. Bivariate phase-rectified signal averaging. Physica 2008; 387(21):5091-5100. ISSN 0378-4371.

[3] Bauer A, Kantelhardt JW, Bunde A, et al. Phase-rectified signal averaging detects quasi-periodicities in nonstationary data. Phys Stat Mech Its Appl 2006;364:423-434.

[4] Bauer A, Morley-Davies A, Barthel P, et al. Bivariate phaserectified signal averaging for assessment of spontaneous baroreflex sensitivity: pilot study of the technology. J Electrocardiol 2020;43(6):649-653.

[5] Castiglioni P, Di Rienzo M, Parati G. How should the baroreflex sensitivity on the heart be estimated? J Electrocardiol 2011;44(3):391-392.

[6] Müller A, Morley-Davies A, Barthel P, et al. Bivariate phase-rectified signal averaging for assessment of spontaneous baroreflex sensitivity: normalization of the results. $\mathbf{J}$ Electrocardiol 2012;45(1):77-81.

[7] Martínez-García P, Lerma C, Infante O. Baroreflex sensitivity estimation by the sequence method with delayed signals. Clin Auton Res 2012;22(6):289-297.

[8] Gouveia S, Rocha AP, Laguna P, et al. Time domain baroreflex sensitivity assessment by joint analysis of spontaneous SBP and RR series. Biomed Signal Process Control 2009; 4:254-261.

[9] Maestri R, La Rovere MT, Raczak G, et al. Estimation of baroreflex sensitivity by the bivariate phase rectified signal averaging method: a comparison with the phenylephrine method. Physiol Meas 2017;38(10):1874-1884.

[10] Goldberger AL, Amaral LAN, Glass L, et al. Physiobank, Physiotoolkit, and Physionet: Components of a new research resource for complex Physiologic Signals. Circulation 2003;101(23):e215-e220.

[11] Heldt T, Oefinger MB, Hoshiyama M, et al. Circulatory response to passive and active changes in posture. Comput Cardiol 2003;30:263-266.

[12] Nemati S, Malhotra A, Clifford GD. Data fusion for improved respiration rate estimation. EURASIP J Adv Signal Process 2010;2010:926305.

[13] Patton DJ, Triedman JK, Perrott MH, et al. Baroreflex gain: characterization using autoregressive moving average analysis. Am J Physiol 1996;270:H1240-9.

Address for correspondence:

Massimo W. Rivolta

Dipartimento di Informatica, Università degli Studi di Milano, Via Celoria 18, 20133, Milan, Italy

massimo.rivolta@unimi.it 